\title{
Comportamentos alimentares de risco à saúde e fatores associados entre motoristas de caminhão
}

\author{
Unhealthy eating habits and associated risk factors among truck \\ drivers
}

Edmarlon Girotto (https://orcid.org/0000-0001-9345-3348) ${ }^{1}$

Mathias Roberto Loch (https://orcid.org/0000-0002-2680-4686) ${ }^{2}$

Arthur Eumann Mesas (https://orcid.org/0000-0002-0088-8607) ${ }^{3}$

Alberto Durán González (https://orcid.org/0000-0002-4203-9400) ${ }^{3}$

Camilo Molino Guidoni (https://orcid.org/0000-0001-5844-143X) ${ }^{1}$

Selma Maffei de Andrade (https://orcid.org/0000-0001-6843-8330) ${ }^{3}$
${ }^{1}$ Departamento de Ciências Farmacêuticas, Universidade Estadual de Londrina (UEL). Av. Robert Kock 60, Operária. 86038-350 Londrina PR Brasil. edmarlon78@gmail.com

${ }^{2}$ Departamento de

Educação Física, UEL. Londrina PR Brasil.

${ }^{3}$ Departamento de Saúde

Coletiva, UEL. Londrina PR Brasil.

\begin{abstract}
The scope of this article is to identify risk factors associated with unhealthy eating habits among truck drivers. It involved a cross-sectional study carried out with truck drivers individually interviewed at the Port of Paranagua in the State of Paraná, Brazil. Information was obtained on the consumption of fruit, vegetables, fried salted food, sweetened beverages, and the removal of visible fat from red meat and of skin from chicken meat. Hierarchical Poisson regression models were applied to identify factors associated with unhealthy eating habits. Of those interviewed ( $n$ $=670$ ), $53.1 \%$ had four or more unhealthy eating habits. This condition was associated with age less than 40 years (prevalence ratio, $P R=1.49 ; 95 \%$ confidence interval, $C I=1.28-1.73)$, moderate, low or very low ability to exercise the profession (PR 1.28; 95\% CI 1.08-1.52), not taking physical exercise in free time $(P R=1.66 ; 95 \% C I=1.38$ 2.00), overall self-reported eating habits as poor or very poor $(R P=1.25$; IC95\% $=1.05-1.49)$ and body mass index $<25 \mathrm{Kg} / \mathrm{m}^{2}(P R=1.22 ; 95 \% \mathrm{CI}$ $=1.05-1.43)$. More than half of the truck drivers revealed unhealthy eating habits, highlighting the need for strategies to rectify these habits.
\end{abstract}

Key words Eating habits, Truck drivers, Working conditions
Resumo $O$ objetivo deste artigo é identificar fatores associados aos comportamentos alimentares de risco à saúde entre motoristas de caminhão. Trata-se de um estudo transversal realizado com motoristas de caminhão entrevistados no Porto de Paranaguá. Foram obtidas informações sobre o consumo de frutas, verduras e legumes, salgados fritos, bebidas açucaradas industrializadas e doces, e retirada de gordura visivel de carne vermelha $e$ da pele de carne de frango. Modelos hierarquizados foram construidos para identificar fatores associados aos comportamentos alimentares de risco à saúde. Dos entrevistados $(n=670), 53,1 \%$ apresentaram quatro ou mais comportamentos alimentares de risco à saúde. Tal condição associou-se com idade inferior a 40 anos $(R P=1,49$; IC95\% = 1,28-1,73), capacidade para exercer a profissão referida como moderada/baixa/muito baixa (RP 1,28; IC 95\% 1,08-1,52), não praticar atividade física no tempo livre ( $R P=1,66$; IC95\% $=1,38-2,00)$, qualidade da alimentação referida como ruim/muito ruim $(R P=1,25 ;$ IC95\% $=$ 1,05-1,49) e índice de massa corporal (IMC) $<25$ $\mathrm{Kg} / \mathrm{m}^{2}(R P=1,22 ; \mathrm{IC} 95 \%=1,05-1,43)$. Mais da metade dos motoristas de caminhão apresentaram comportamentos alimentares de risco à saúde, reforçando a necessidade de estratégias de incentivo à redução desses hábitos.

Palavras-chave Comportamento alimentar, Motoristas de caminhão, Condições de trabalho 


\section{Introdução}

Nas últimas décadas, tem-se percebido alterações no perfil epidemiológico e nutricional de diversas populações, decorrentes de alterações em inúmeros aspectos ambientais, sociais e comportamentais, incluindo mudanças nos hábitos alimentares ${ }^{1,2}$. Entre essas mudanças, destacamse o aumento do consumo de alimentos industrializados e a redução do consumo de frutas, legumes e verduras ${ }^{3}$, o que influencia diretamente no aumento da prevalência de doenças crônicas não transmissíveis ${ }^{4,5}$. As alterações mencionadas extrapolam a população geral e certos grupos populacionais, como motoristas de caminhão, podem ser mais susceptíveis aos efeitos deletérios dessas mudanças.

Os motoristas de caminhão representam uma categoria profissional de suma importância para a economia do Brasil, pois são responsáveis pelo transporte da maior parte das matérias-primas e produtos manufaturados. Dados da Confederação Nacional dos Transportes (CNT) demonstram que o transporte rodoviário representa cerca de $60 \%$ da matriz de transporte de cargas no Brasil $^{6}$.

Apesar da sua importância no contexto econômico, os motoristas de caminhão são, muitas vezes, expostos a condições precárias de traba$\mathrm{lho}^{7}$, o que inclui longas jornadas de trabalho ${ }^{8}$, turnos irregulares ${ }^{8}$, ausência de pausas para descanso $^{8}$, estrutura rodoviária precária ${ }^{9}$, risco de assaltos e acidentes de trânsito ${ }^{10}$.

As repercussões dos aspectos mencionados podem prejudicar a saúde desses trabalhadores, destacando-se o excesso de peso ${ }^{11}$, hipertensão arterial, diabetes mellitus ${ }^{12}$, distúrbios do sono ${ }^{11,13-}$ ${ }^{15}$, estresse ${ }^{11,13,14} \mathrm{e}$ fadiga ${ }^{11,13}$. Ademais, o sedentarismo ${ }^{16}$ e o aumento de condutas alimentares de risco à saúde ${ }^{11,17}$ podem ser mais prevalentes nessa categoria profissional em relação à população geral.

No Brasil, estudo descritivo com 470 motoristas de caminhão, o qual não analisou diferenças estatísticas entre os grupos, verificou que aqueles que trabalhavam à noite apresentaram maior consumo de carnes, óleos e gorduras, quando comparado aos que trabalhavam durante o dia, situação atribuída ao fato de os trabalhadores do turno da noite enfrentarem dificuldades de adaptação fisiológica e de integração social ${ }^{17}$. Ainda assim, os resultados desse estudo ${ }^{17}$ denotam que aspectos relacionados às condições de trabalho podem interferir nos hábitos alimentares. Em outro estudo brasileiro, realizado em pontos de parada com 400 motoristas de caminhão, identificou-se que $33,3 \%$ frequentemente faziam uso de alimentos gordurosos, e apenas $15,5 \%$ referiam nunca ingerir esses alimentos ${ }^{11}$.

Pesquisadores de outros países propõem intervenções alimentares nesse grupo populacional $^{18,19}$, demonstrando que o uso de tecnologias ${ }^{19}$ e educação presencial e à distância ${ }^{18}$ contribuem para a melhoria de hábitos alimentares. Todavia, essas intervenções somente se justificam e podem ser realizadas com sucesso com o conhecimento adequado dos comportamentos alimentares e de fatores associados, incluindo os ocupacionais, que predispõem esses profissionais a comportamentos não saudáveis. Por conseguinte, estas informações são incipientes no Brasil. Desta forma, este estudo tem como objetivo caracterizar os comportamentos alimentares e identificar fatores associados aos comportamentos alimentares de risco à saúde entre motoristas de caminhão.

\section{Métodos}

Trata-se de um estudo transversal com motoristas de caminhão que se encontravam estacionados no mês de julho de 2012 no Pátio de Triagem do Porto de Paranaguá, Paraná, Brasil. O Porto de Paranaguá é um dos principais corredores de exportação do Brasil e o maior porto graneleiro da América Latina. Os caminhões que realizam o transporte de grãos até o Porto de Paranaguá devem obrigatoriamente passar pelo Pátio de Triagem, onde se avalia a qualidade do produto transportado e também se aguarda o momento de descarga do caminhão.

Para o cálculo do tamanho amostral necessário para analisar os fatores associados aos comportamentos alimentares de risco à saúde, consideraram-se os seguintes parâmetros: comportamento alimentar de risco à saúde em torno de $50 \%$, variação dessa frequência segundo diferentes categorias das variáveis estudadas estimada em $6 \%$ ou mais ${ }^{20}$. Admitindo-se $5 \%$ de erro alfa e $20 \%$ de erro beta, calculou-se o número mínimo de 623 participantes. A seleção dos motoristas participantes foi por conveniência, uma vez que o fluxo de veículos e o estacionamento de caminhões no pátio ocorriam de forma não sistemática. Realizou-se estudo piloto no Pátio do Porto de Paranaguá para avaliar o fluxo de caminhões, identificar os pontos para a realização da coleta de dados, testar os instrumentos de pesquisa e definir estratégia de abordagem dos motoristas. 
A coleta de dados foi realizada por pesquisadores devidamente treinados e ocorreu entre $8 \mathrm{~h}$ e $18 \mathrm{~h}$ durante sete dias consecutivos do mês de julho de 2012. Não foram adotados critérios de exclusão para a execução deste estudo. Foram obtidas, por meio de entrevista, informações sobre características sociodemográficas, de estilo de vida, comportamentais e ocupacionais e relativas ao consumo alimentar dos motoristas de caminhão. Para a construção do instrumento de coleta de dados, em especial das questões sobre os hábitos de vida, baseou-se no instrumento utilizado no Estudo Vigitel 2011, o qual teve por objetivo monitorar a frequência e a distribuição dos principais determinantes das doenças crônicas não transmissíveis por inquérito telefônico ${ }^{21}$. Especificamente para os hábitos alimentares, adaptouse o instrumento do Estudo Vigitel $2011^{21}$, utilizando-se como respostas escala de frequência ou ocorrência (de "nunca" a "sempre").

$\mathrm{O}$ desfecho analisado neste estudo foram os comportamentos alimentares de risco à saúde. Para isso, avaliaram-se quatro comportamentos alimentares positivos (consumo de frutas; consumo de verduras e legumes; retirada de gordura antes de comer carne vermelha; e retirada da pele antes de comer frango) e três comportamentos negativos (consumo de salgados fritos; consumo de bebidas açucaradas industrializadas; e consumo de doces). Esses sete comportamentos alimentares foram obtidos por meio de entrevista, e a resposta para cada um dos comportamentos poderia ser: "nunca/raramente"; "às vezes"; "quase sempre/sempre". Foram considerados comportamentos de risco à saúde caso o entrevistado respondesse "nunca/raramente" ou "às vezes" para um comportamento positivo ou se respondesse "quase sempre/sempre" ou "às vezes" para um comportamento negativo. Assim, o motorista poderia apresentar de zero a sete comportamentos de risco à saúde, os quais, para análise exploratória, foram categorizados em: "até três" e "quatro ou mais".

As variáveis independentes analisadas foram estratificadas em três níveis: Nível Distal (faixa etária, viver com companheira, escolaridade, cor da pele autorreferida, acesso a plano de saúde); Nível Intermediário (renda como motorista, distância do último local de carregamento, característica salarial, horas de trabalho ininterruptas, direção do veículo estando bastante cansado, turno irregular de trabalho, percepção sobre a capacidade para exercer a profissão); Nível Proximal [atividade física no tempo livre, consumo excessivo de bebidas alcoólicas, qualidade da ali- mentação autorreferida, tabagismo atual, índice de massa corporal (IMC)]. Foi considerado turno irregular quando o motorista referisse exercer a profissão tanto no turno noturno quanto no diurno de forma semelhante. A atividade física no tempo livre foi considerada positiva independente da frequência ou duração referida pelo motorista. Considerou-se como abusivo o consumo de mais de cinco doses de bebida alcoólica pelos motoristas em uma mesma ocasião nos últimos 30 dias. O IMC foi calculado com as medidas de peso e altura autorreferidas, de acordo com a fórmula IMC $=$ peso $(\mathrm{kg}) /$ altura $^{2}(\mathrm{~m})$. Os pontos de corte de IMC adotados foram: baixo peso (IMC $\left.<18,5 \mathrm{Kg} / \mathrm{m}^{2}\right)$; eutrofia (IMC 18,5-24,99 Kg/m ${ }^{2}$ ); sobrepeso (IMC $25-29,99 \mathrm{Kg} / \mathrm{m}^{2}$ ) e obesidade (IMC e > 30,00 Kg/m²). A seleção das variáveis para cada nível de determinação (distal, intermediário e proximal) se baseou na relação hierárquica (temporal e causal) existente entre estas variáveis ${ }^{22}$, conforme esquema apresentado na Figura 1. Neste caso, variáveis socioeconômicas e demográficas foram enquadradas como variáveis distais; condições de trabalho como intermediárias; e hábitos de vida como variáveis proximais. Acesso a plano de saúde foi considerada uma variável distal por refletir condições socioeconômicas. Apesar de a renda como motorista poder ser considerada também uma variável socioeconômica, considerou-se, para o presente estudo, que esta representaria as condições de trabalho do motorista de caminhão de forma mais específica, sendo, portanto, incluída no bloco das variáveis intermediárias.

Os dados foram duplamente digitados por pesquisadores distintos em banco de dados elaborado no programa Epi Info $^{\circledR}$, versão 3.5.3, para Windows ${ }^{\circledR}$, e comparados para correções de eventuais erros de digitação. A análise foi realizada utilizando o programa Statistical Package for the Social Sciences (SPSS), versão 19.0 para Windows ${ }^{\circledR}$. A análise dos dados ocorreu em duas etapas: análise univariada e multivariada hierarquizada. As associações entre as variáveis independentes e o desfecho "quatro ou mais comportamentos alimentares de risco à saúde" foram verificadas utilizando a Regressão de Poisson com variância robusta, com estimativa da Razão de Prevalência (RP) e do intervalo de confiança de $95 \%$ (IC 95\%).

$\mathrm{Na}$ análise univariada (não ajustada) verificou-se associação entre o desfecho e cada variável independente testada. Em seguida, construíramse modelos de regressão multivariada hierarquizada, incluindo-se as variáveis independentes 


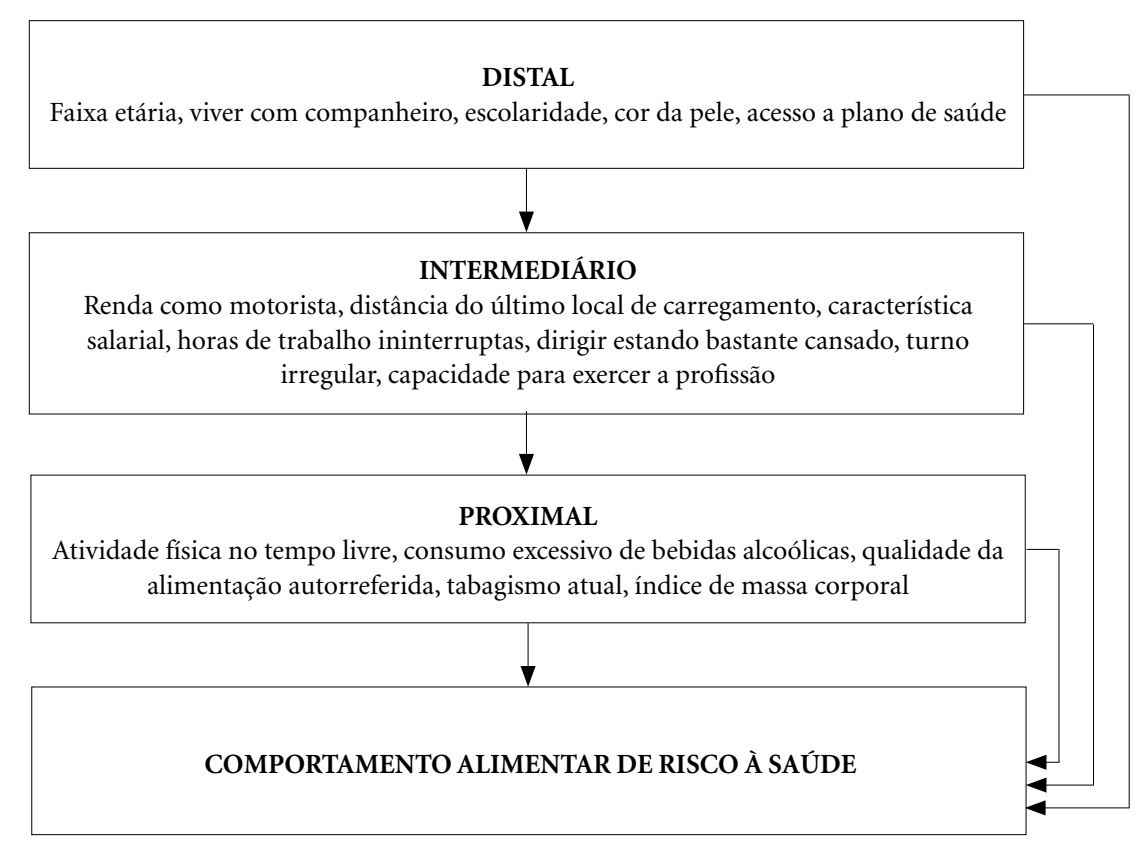

Figura 1. Modelo hierárquico conceitual dos fatores determinantes de comportamentos alimentares de risco à saúde.

compreendidas em cada nível que apresentaram $\mathrm{p}<0,20$ na etapa anterior ${ }^{22}$. A análise multivariada hierarquizada iniciou-se com a inclusão das variáveis do nível distal. As variáveis que neste modelo apresentaram $\mathrm{p}<0,10$ foram incluídas no modelo subsequente, o qual incluiu as variáveis do nível intermediário. Por fim, seguiu-se o mesmo processo para as variáveis proximais. Nos resultados foram apresentadas apenas as variáveis que apresentaram $\mathrm{p}<0,05$ no modelo final hierarquizado.

A presente investigação foi aprovada pelo Comitê de Ética em Pesquisa da Universidade Estadual de Londrina (UEL). Todos os motoristas foram informados quanto aos objetivos da pesquisa e, após concordância e assinatura de um Termo de Consentimento Livre e Esclarecido, responderam às questões da entrevista.

\section{Resultados}

Durante o período de coleta de dados, 9.486 caminhões ingressaram no Pátio de Triagem do Porto de Paranaguá, sendo abordados 773 motoristas, dos quais $98(12,7 \%)$ recusaram-se a participar do estudo. Dos 675 motoristas que iniciaram a entrevista, cinco foram chamados para descarregar seus veículos durante a realização da entrevista e não forneceram todas as informações necessárias. Dessa forma, compuseram este estudo 670 motoristas de caminhão. Os motoristas, todos do sexo masculino, apresentaram idade média de 41,9 anos (Desvio-Padrão [DP] = 11,1), mínima de 21 e máxima de 75 anos. A renda mensal como motorista variou de $\mathrm{R} \$ 800,00$ a $\mathrm{R} \$ 25.000,00$, com média de $\mathrm{R} \$ 2.932,96(\mathrm{DP}=\mathrm{R} \$ 1.972,40)$.

A maioria dos motoristas referiu viver com companheira $(79,0 \%)$, ser branco $(66,4 \%)$ e não possuir plano de saúde $(70,3 \%)$. Verificou-se que $89,4 \%$ tinham renda baseada na produtividade e $80,0 \%$ renda inferior a $\mathrm{R} \$ 3.000,00$. Cerca de $25,0 \%$ referiram turno de trabalho irregular. Destaca-se, ainda, que a maior parte relatou não realizar atividade física no tempo livre $(68,2 \%)$, tinha sobrepeso ou obesidade $(81,1 \%)$ e apenas $8,8 \%$ consideravam sua alimentação ruim ou muito ruim (Tabela 1 ).

Observa-se a distribuição dos comportamentos segundo as variáveis independentes avaliadas. 
Tabela 1. Distribuição dos motoristas de caminhão de acordo com as variáveis independentes avaliadas segundo comportamentos alimentares positivos e negativos, Porto de Paranaguá, Paraná, Brasil, 2012 (n=670).

\begin{tabular}{|c|c|c|c|c|c|c|c|c|}
\hline \multirow{3}{*}{ Variáveis Independentes } & \multirow{3}{*}{ n $(\%)$} & \multicolumn{7}{|c|}{ Comportamentos Alimentares de Risco à Saúde } \\
\hline & & \multicolumn{4}{|c|}{$\begin{array}{l}\text { Positivos: } \\
\text { Frequência de nunca/raramente/às vezes }\end{array}$} & \multicolumn{3}{|c|}{$\begin{array}{c}\text { Negativos: } \\
\text { Frequência de sempre/quase } \\
\text { sempre/sempre }\end{array}$} \\
\hline & & $\begin{array}{c}1 \\
\mathbf{n}(\%)\end{array}$ & $\begin{array}{c}2 \\
\text { n }(\%)\end{array}$ & $\begin{array}{c}3 \\
\text { n }(\%)\end{array}$ & $\begin{array}{c}4 \\
\mathrm{n}(\%)\end{array}$ & $\begin{array}{c}5 \\
\mathbf{n}(\%)\end{array}$ & $\begin{array}{c}6 \\
\mathrm{n}(\%) \\
\end{array}$ & $\begin{array}{c}7 \\
\text { n }(\%)\end{array}$ \\
\hline \multicolumn{9}{|l|}{ Variáveis Distais } \\
\hline \multicolumn{9}{|l|}{ Faixa etária } \\
\hline Menos de 40 anos & $314(46,9)$ & $199(63,4)$ & $93(29,6)$ & $200(63,7)$ & $162(51,6)$ & $179(57,0)$ & $264(84,1)$ & $204(65,0)$ \\
\hline 40 anos ou mais & $356(53,1)$ & $169(47,5)$ & $81(22,8)$ & $184(51,7)$ & $146(41,0)$ & $148(41,6)$ & $245(68,8)$ & $196(55,1)$ \\
\hline \multicolumn{9}{|l|}{ Vive com companheira } \\
\hline Sim & $567(84,6)$ & $308(54,3)$ & $146(25,7)$ & $314(55,4)$ & $259(45,7)$ & $275(48,5)$ & $423(74,6)$ & $334(58,9)$ \\
\hline Não & $103(15,4)$ & $60(58,3)$ & $28(27,2)$ & $70(68,0)$ & $49(47,6)$ & $52(50,5)$ & $86(83,5)$ & $66(64,1)$ \\
\hline \multicolumn{9}{|l|}{ Escolaridade } \\
\hline Oito anos ou menos & $280(41,8)$ & $149(53,2)$ & $78(27,9)$ & $164(58,6)$ & $122(43,6)$ & $131(46,8)$ & $221(78,9)$ & $183(65,4)$ \\
\hline Mais de oito anos & $390(58,2)$ & $219(56,2)$ & $96(24,6)$ & $220(56,4)$ & $186(47,7)$ & $196(50,3)$ & $288(73,8)$ & $217(55,6)$ \\
\hline \multicolumn{9}{|l|}{ Cor da pele } \\
\hline Branca & $445(66,4)$ & $232(52,1)$ & $113(25,4)$ & $251(56,4)$ & $201(45,2)$ & $219(49,2)$ & $342(76,9)$ & $253(56,9)$ \\
\hline Não branca & $225(33,6)$ & $136(60,4)$ & $61(27,1)$ & $133(59,1)$ & $107(47,6)$ & $108(48,0)$ & $167(74,2)$ & $147(65,3)$ \\
\hline \multicolumn{9}{|l|}{ Acesso a plano de saúde } \\
\hline Sim & $199(29,7)$ & $98(49,1)$ & $54(27,1)$ & $114(57,3)$ & $95(47,7)$ & $94(47,2)$ & $151(75,9)$ & $118(59,3)$ \\
\hline Não & $471(70,3)$ & $270(57,3)$ & $120(25,5)$ & $270(57,3)$ & $213(45,2)$ & $233(49,5)$ & $358(76,0)$ & $282(59,9)$ \\
\hline \multicolumn{9}{|l|}{ Variáveis Intermediárias } \\
\hline \multicolumn{9}{|l|}{ Renda como motorista } \\
\hline Menos de $\mathrm{R} \$ 3.000,00$ & $536(80,0)$ & $303(56,5)$ & $141(26,3)$ & $312(58,2)$ & $248(46,3)$ & $267(49,8)$ & $407(75,9)$ & $321(59,9)$ \\
\hline $\mathrm{R} \$ 3.000,00$ ou mais & $134(20,0)$ & $65(48,5)$ & $33(24,6)$ & $72(53,7)$ & $60(44,8)$ & $60(44,8)$ & $102(76,1)$ & $79(59,0)$ \\
\hline \multicolumn{9}{|l|}{$\begin{array}{l}\text { Distância do último local } \\
\text { de carregamento }\end{array}$} \\
\hline Menos $1200 \mathrm{~km}$ & $476(71,0)$ & $254(53,4)$ & $126(26,5)$ & $278(58,4)$ & $220(46,2)$ & $86(44,3)$ & $358(75,2)$ & $278(58,4)$ \\
\hline $1200 \mathrm{~km}$ ou mais & $194(29,0)$ & $114(58,8)$ & $48(24,7)$ & $106(54,6)$ & $88(45,4)$ & $241(50,6)$ & $151(77,8)$ & $122(62,9)$ \\
\hline \multicolumn{9}{|l|}{ Característica salarial } \\
\hline Fixo & $71(10,6)$ & $37(52,1)$ & $24(33,8)$ & $43(60,6)$ & $36(50,7)$ & $39(54,9)$ & $52(73,2)$ & $52(73,2)$ \\
\hline $\begin{array}{l}\text { Fixo/produtividade ou } \\
\text { produtividade }\end{array}$ & $599(89,4)$ & $331(55,3)$ & $150(25,0)$ & $341(56,9)$ & $272(45,4)$ & $288(48,1)$ & $457(76,3)$ & $348(58,1)$ \\
\hline \multicolumn{9}{|l|}{$\begin{array}{l}\text { Horas de trabalho } \\
\text { ininterruptas }\end{array}$} \\
\hline Mais de oito horas & $57(8,5)$ & $37(64,9)$ & $16(28,1)$ & $28(49,1)$ & $24(42,1)$ & $31(54,4)$ & $46(80,7)$ & $30(52,6)$ \\
\hline Oito horas ou menos & $613(91,5)$ & $331(54,0)$ & $158(25,8)$ & $356(58,1)$ & $284(46,3)$ & $296(48,3)$ & $463(75,5)$ & $370(60,4)$ \\
\hline \multicolumn{9}{|l|}{$\begin{array}{l}\text { Direção do veículo } \\
\text { estando bastante cansado }\end{array}$} \\
\hline Sim & $59(8,8)$ & $40(67,8)$ & $22(37,3)$ & $31(52,5)$ & $26(44,1)$ & $35(59,3)$ & $51(86,4)$ & $34(57,6)$ \\
\hline Não & $611(91,2)$ & $328(53,7)$ & $152(24,9)$ & $353(57,8)$ & $282(46,2)$ & $292(47,8)$ & $458(75,0)$ & $366(59,9)$ \\
\hline \multicolumn{9}{|l|}{ Turno irregular } \\
\hline Sim & $171(25,5)$ & $100(58,5)$ & $45(26,3)$ & $99(57,9)$ & $77(45,0)$ & $96(56,1)$ & $141(82,5)$ & $104(60,8)$ \\
\hline Não & $499(74,5)$ & $268(53,7)$ & $129(25,9)$ & $285(57,1)$ & $231(46,3)$ & $231(46,3)$ & $368(73,7)$ & $296(59,3)$ \\
\hline \multicolumn{9}{|l|}{$\begin{array}{l}\text { Capacidade para exercer } \\
\text { a profissão }\end{array}$} \\
\hline Muito boa ou boa & $594(88,7)$ & $320(53,9)$ & $152(25,6)$ & $334(56,2)$ & $268(45,1)$ & $290(48,8)$ & $455(76,6)$ & $353(59,4)$ \\
\hline $\begin{array}{l}\text { Moderada ou baixa ou } \\
\text { muito baixa }\end{array}$ & $76(11,3)$ & $48(63,2)$ & $22(28,9)$ & $50(65,8)$ & $40(52,6)$ & $37(48,7)$ & $54(71,1)$ & $47(61,8)$ \\
\hline
\end{tabular}


Tabela 1. Distribuição dos motoristas de caminhão de acordo com as variáveis independentes avaliadas segundo comportamentos alimentares positivos e negativos, Porto de Paranaguá, Paraná, Brasil, 2012 (n=670).

\begin{tabular}{|c|c|c|c|c|c|c|c|c|}
\hline \multirow{3}{*}{ Variáveis Independentes } & \multirow{3}{*}{ n (\%) } & \multicolumn{7}{|c|}{ Comportamentos Alimentares de Risco à Saúde } \\
\hline & & \multicolumn{4}{|c|}{$\begin{array}{l}\text { Positivos: } \\
\text { Frequência de nunca/raramente/às vezes }\end{array}$} & \multicolumn{3}{|c|}{$\begin{array}{c}\text { Negativos: } \\
\text { Frequência de sempre/quase } \\
\text { sempre/sempre }\end{array}$} \\
\hline & & $\begin{array}{c}1 \\
\text { n }(\%)\end{array}$ & $\begin{array}{c}2 \\
\text { n }(\%)\end{array}$ & $\begin{array}{c}3 \\
\mathbf{n}(\%) \\
\end{array}$ & $\begin{array}{c}4 \\
\mathrm{n}(\%) \\
\end{array}$ & $\begin{array}{c}5 \\
\mathbf{n}(\%) \\
\end{array}$ & $\begin{array}{c}6 \\
\mathbf{n}(\%) \\
\end{array}$ & $\begin{array}{c}7 \\
\mathbf{n}(\%) \\
\end{array}$ \\
\hline \multicolumn{9}{|l|}{ Variáveis Proximais } \\
\hline \multicolumn{9}{|l|}{$\begin{array}{l}\text { Atividade física no } \\
\text { tempo livre }\end{array}$} \\
\hline Sim & $213(31,8)$ & $76(35,7)$ & $30(14,1)$ & $102(47,9)$ & $78(36,6)$ & $94(44,1)$ & $156(73,2)$ & $124(58,2)$ \\
\hline Não & $457(68,2)$ & $292(63,9)$ & $144(31,5)$ & $282(61,7)$ & $230(50,3)$ & $233(51,0)$ & $353(77,2)$ & $276(60,4)$ \\
\hline \multicolumn{9}{|l|}{$\begin{array}{l}\text { Consumo excessivo de } \\
\text { bebidas alcoólicas }\end{array}$} \\
\hline Sim & $195(29,1)$ & $119(61,0)$ & $47(24,1)$ & $124(63,6)$ & $99(50,8)$ & $119(61,0)$ & $151(77,4)$ & $109(55,9)$ \\
\hline Não & $475(70,9)$ & $249(52,4)$ & $127(26,7)$ & $260(54,7)$ & $209(44,0)$ & $208(43,8)$ & $358(75,4)$ & $291(61,3)$ \\
\hline \multicolumn{9}{|l|}{$\begin{array}{l}\text { Qualidade da } \\
\text { alimentação } \\
\text { autorreferida }\end{array}$} \\
\hline $\begin{array}{l}\text { Muito boa ou boa ou } \\
\text { regular }\end{array}$ & $611(91,2)$ & $321(52,5)$ & $149(24,4)$ & $346(56,6)$ & $275(45,0)$ & $287(47,0)$ & $459(75,1)$ & $360(58,9)$ \\
\hline Ruim ou muito ruim & $59(8,8)$ & $47(79,7)$ & $25(42,4)$ & $38(64,4)$ & $33(55,9)$ & $40(67,8)$ & $50(84,7)$ & $40(67,8)$ \\
\hline \multicolumn{9}{|l|}{ Tabagismo atual } \\
\hline Sim & $169(25,2)$ & $93(55,0)$ & $46(27,2)$ & $110(65,1)$ & $83(49,1)$ & $82(48,5)$ & $118(69,8)$ & $103(60,9)$ \\
\hline Não & $501(74,8)$ & $275(54,9)$ & $128(25,5)$ & $274(54,7)$ & $225(44,9)$ & $245(48,9)$ & $391(78,0)$ & $297(59,3)$ \\
\hline \multicolumn{9}{|l|}{$\begin{array}{l}\text { Índice de massa corporal } \\
(\mathrm{IMC})^{\star}\end{array}$} \\
\hline $\begin{array}{l}\text { Baixo peso ou } \\
\text { eutrófico }\end{array}$ & $126(18,9)$ & $72(57,1)$ & $33(26,2)$ & $84(66,7)$ & $63(50,0)$ & $63(50,0)$ & $107(84,9)$ & $85(67,5)$ \\
\hline $\begin{array}{l}\text { Sobrepeso ou } \\
\text { obesidade }\end{array}$ & $542(81,1)$ & $296(54,4)$ & $141(25,9)$ & $300(55,1)$ & $245(45,0)$ & $264(48,5)$ & $402(73,9)$ & $315(57,9)$ \\
\hline
\end{tabular}

Destaca-se que motoristas mais jovens (menos de 40 anos) apresentaram maiores frequências de todos os comportamentos avaliados. Os motoristas que referiram dirigir estando bastante cansados relataram maior frequência dos seguintes comportamentos alimentares de risco à saúde: maior consumo de bebidas açucaradas industrializadas e menor consumo de frutas, verduras e legumes. Motoristas que referiram qualidade da alimentação ruim ou muito ruim também reportaram menor consumo de frutas, verduras e legumes e maior consumo de salgados fritos e de bebidas açucaradas industrializadas. Motoristas com sobrepeso ou obesidade relataram menor consumo de doces e de bebidas açucaradas industrializadas e maior frequência de retirada da gordura da carne vermelha para o consumo (Tabela 1).
Dos motoristas avaliados, o principal comportamento positivo observado foi o consumo de verduras e legumes. Em relação aos comportamentos negativos, destacou-se o consumo de bebidas açucaradas industrializadas (Figura 2). Vale ressaltar que $53,1 \%$ apresentaram quatro ou mais comportamentos alimentares de risco à saúde.

$\mathrm{Na}$ análise não ajustada (Tabela 2), a idade inferior a 40 anos (Nível Distal), dirigir estando bastante cansado, dirigir em turno irregular, menor capacidade para exercer a profissão (Nível Intermediário), não praticar atividade física no tempo livre, consumir excessivamente bebidas alcoólicas, percepção de qualidade da alimentação ruim ou muito ruim e categoria de IMC relacionada a baixo peso ou eutrófico (Nível Proximal) mostraram-se associadas a "quatro ou mais comportamentos alimentares de risco à saúde”. 


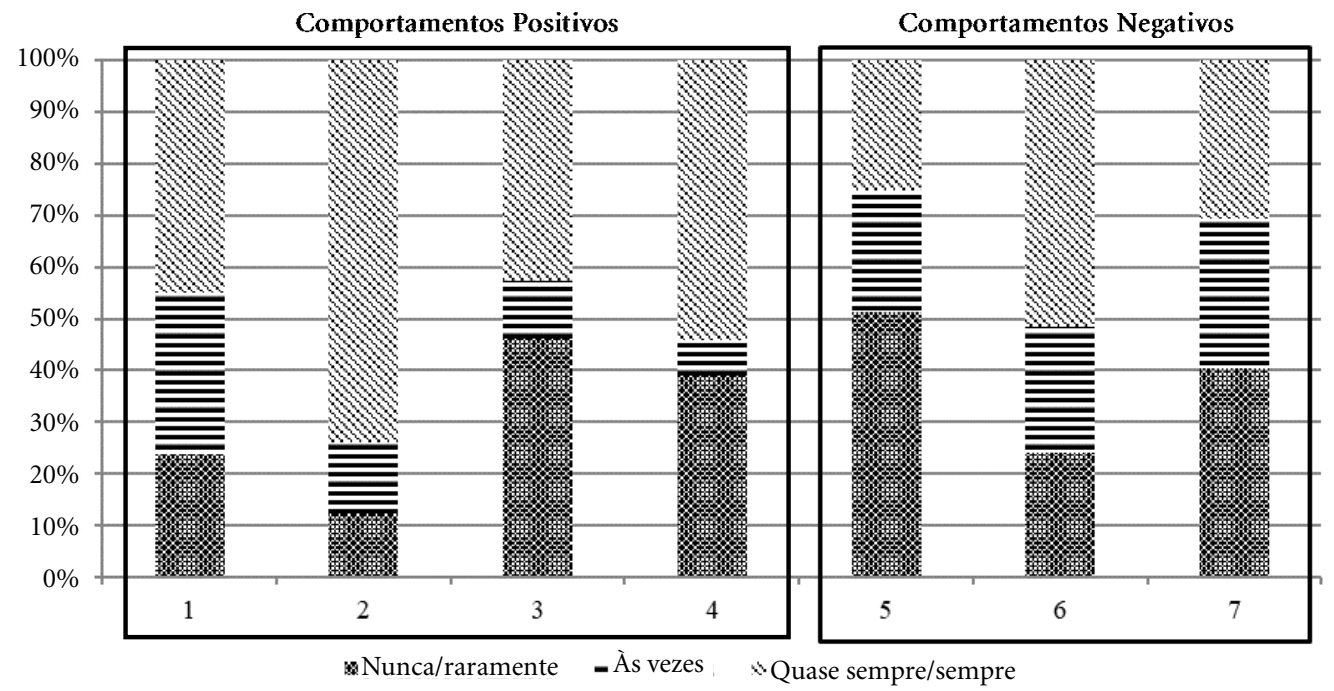

1 - Consumo de frutas; 2 - Consumo de verduras e legumes; 3 - Retira gordura antes de comer carne vermelha; 4 - Retira pele antes de comer carne de frango; 5 - Consumo de salgados fritos; 6 - Consumo de bebidas açucaradas industrializadas; 7 - Consumo de doces.

Figura 2. Distribuição dos motoristas de caminhão segundo frequência dos comportamentos (negativos e positivos) avaliados, Porto de Paranaguá, Paraná, Brasil, $2012(\mathrm{n}=670)$.

Tabela 2. Associação entre comportamentos alimentares de risco à saúde ( $\geq 4$ comportamentos) e variáveis independentes (análise não-ajustada), Porto de Paranaguá, Paraná, Brasil, 2012.

\begin{tabular}{|c|c|c|c|}
\hline \multirow{3}{*}{ Variáveis independentes } & \multicolumn{3}{|c|}{ Comportamentos alimentares de risco à saúde } \\
\hline & \multicolumn{3}{|c|}{$\geq 4$ comportamentos } \\
\hline & n (\%) & RP (IC 95\%) & Valor $\mathbf{p}$ \\
\hline \multicolumn{4}{|l|}{ Variáveis Distais } \\
\hline \multicolumn{4}{|l|}{ Faixa etária } \\
\hline Menos de 40 anos & $208(66,2)$ & $1,59(1,38-1,84)$ & $<0,001$ \\
\hline 40 anos ou mais & $148(41,6)$ & 1,00 & \\
\hline \multicolumn{4}{|l|}{ Vive com companheira } \\
\hline Sim & $298(52,5)$ & 1,00 & 0,470 \\
\hline Não & $58(56,3)$ & $1,07(0,89-1,29)$ & \\
\hline \multicolumn{4}{|l|}{ Escolaridade } \\
\hline Oito anos ou menos & $154(55,0)$ & $1,06(0,92-1,23)$ & 0,410 \\
\hline Mais de oito anos & $202(51,8)$ & 1,00 & \\
\hline \multicolumn{4}{|l|}{ Cor da pele } \\
\hline Branca & $226(50,8)$ & 1,00 & \\
\hline Não branca & $130(57,8)$ & $1,14(0,99-1,31)$ & 0,080 \\
\hline \multicolumn{4}{|l|}{ Acesso a plano de saúde } \\
\hline Sim & $102(51,3)$ & 1,00 & \\
\hline Não & $254(53,9)$ & $1,05(0,90-1,23)$ & 0,531 \\
\hline \multicolumn{4}{|l|}{ Variáveis Intermediárias } \\
\hline \multicolumn{4}{|l|}{ Renda como motorista } \\
\hline Menos de $\mathrm{R} \$ 3.000,00$ & $290(54,1)$ & $1,10(0,91-1,33)$ & 0,329 \\
\hline $\mathrm{R} \$ 3.000,00$ ou mais & $66(49,3)$ & 1,00 & \\
\hline
\end{tabular}


Tabela 2. Associação entre comportamentos alimentares de risco à saúde ( $\geq 4$ comportamentos) e variáveis independentes (análise não-ajustada), Porto de Paranaguá, Paraná, Brasil, 2012.

\begin{tabular}{|c|c|c|c|}
\hline \multirow{3}{*}{ Variáveis independentes } & \multicolumn{3}{|c|}{ Comportamentos alimentares de risco à saúde } \\
\hline & \multicolumn{3}{|c|}{$\geq 4$ comportamentos } \\
\hline & n (\%) & RP (IC 95\%) & Valor $\mathbf{p}$ \\
\hline \multicolumn{4}{|l|}{ Distância do último local de carregamento } \\
\hline Menos $1200 \mathrm{~km}$ & $254(53,4)$ & $1,02(0,76-1,19)$ & 0,854 \\
\hline $1200 \mathrm{~km}$ ou mais & $102(52,6)$ & 1,00 & \\
\hline \multicolumn{4}{|l|}{ Característica salarial } \\
\hline Fixo & $42(59,2)$ & $1,13(0,92-1,39)$ & 0,254 \\
\hline Fixo/produtividade ou produtividade & $314(52,4)$ & 1,00 & \\
\hline \multicolumn{4}{|l|}{ Horas de trabalho ininterruptas } \\
\hline Mais de oito horas & $31(54,4)$ & $1,03(0,80-1,32)$ & 0,841 \\
\hline Oito horas ou menos & $325(53,0)$ & & \\
\hline \multicolumn{4}{|l|}{ Direção do veículo estando bastante cansado } \\
\hline Sim & $38(64,4)$ & $1,24(1,01-1,52)$ & 0,041 \\
\hline Não & $318(52,0)$ & 1,00 & \\
\hline \multicolumn{4}{|l|}{ Turno irregular } \\
\hline Sim & $104(60,8)$ & $1,20(1,04-1,40)$ & 0,014 \\
\hline Não & $252(50,4)$ & 1,00 & \\
\hline \multicolumn{4}{|l|}{ Capacidade para exercer a profissão } \\
\hline Muito boa ou boa & $307(51,7)$ & 1,00 & \\
\hline Moderada, baixa ou muito baixa & $49(64,5)$ & $1,25(1,04-1,50)$ & 0,019 \\
\hline \multicolumn{4}{|l|}{ Variáveis Proximais } \\
\hline \multicolumn{4}{|l|}{ Atividade física no tempo livre } \\
\hline Sim & $77(36,2)$ & 1,00 & \\
\hline Não & $279(61,1)$ & $1,69(1,39-2,05)$ & $<0,001$ \\
\hline \multicolumn{4}{|l|}{ Consumo excessivo de bebidas alcoólicas } \\
\hline Sim & $115(59,0)$ & $1,16(1,01-1,35)$ & 0,045 \\
\hline Não & $241(50,7)$ & 1,00 & \\
\hline \multicolumn{4}{|l|}{ Qualidade da alimentação autorreferida } \\
\hline Muito boa ou boa ou regular & $313(51,2)$ & 1,00 & \\
\hline Ruim ou muito ruim & $43(72,9)$ & $1,42(1,20-1,69)$ & $<0,001$ \\
\hline \multicolumn{4}{|l|}{ Tabagismo atual } \\
\hline Sim & $97(57,4)$ & $1,11(0,95-1,30)$ & 0,186 \\
\hline Não & $259(51,7)$ & 1,00 & \\
\hline \multicolumn{4}{|l|}{ Índice de massa corporal } \\
\hline Baixo peso ou eutrófico & $79(62,7)$ & $1,23(1,05-1,45)$ & 0,010 \\
\hline Sobrepeso ou obesidade & $277(50,9)$ & 1,00 & \\
\hline
\end{tabular}

RP: Razão de Prevalência; IC 95\%: Intervalo de confiança 95\%.

Após análise ajustada das variáveis por meio de regressão hierarquizada, conforme modelo teórico conceitual de determinação do desfecho em estudo, mantiveram-se associadas a apresentar "quatro ou mais comportamentos alimentares de risco à saúde": idade inferior a 40 anos (RP 1,49; IC 95\% 1,28-1,73) (Nível Distal), capacidade para exercer a profissão moderada, baixa ou muito baixa (RP 1,28; IC 95\% 1,08-1,52) (Nível Intermediário), não praticar atividade física no tempo livre (RP 1,66; IC 95\% 1,38-2,00), qua- lidade da alimentação referida como ruim ou muito ruim (RP 1,25; IC 95\% 1,05-1,49) e IMC $<25 \mathrm{Kg} / \mathrm{m}^{2}$ (RP 1,22; IC 95\% 1,05-1,43) (Nível Proximal) (Tabela 3$)$.

\section{Discussão}

Os motoristas de caminhão avaliados apresentaram, de forma geral, comportamentos alimentares contraditórios, com níveis de consumo de 
Tabela 3. Associação entre comportamentos alimentares de risco à saúde e variáveis independentes (Análise Hierarquizada), Porto de Paranaguá, Paraná, Brasil, 2012.

\begin{tabular}{|c|c|c|}
\hline \multirow{2}{*}{ Variáveis independentes } & \multicolumn{2}{|c|}{$\begin{array}{l}\text { Quatro ou mais comportamentos alimentares de risco } \\
\text { à saúde }\end{array}$} \\
\hline & PD (IC 050\%) & Valorn \\
\hline \multicolumn{3}{|l|}{ Variáveis Distais } \\
\hline \multicolumn{3}{|l|}{ Faixa etária } \\
\hline Menos de 40 anos & $1,49(1,28-1,73)$ & $<0,001$ \\
\hline 40 anos ou mais & 1,00 & \\
\hline \multicolumn{3}{|l|}{ Variáveis Intermediárias } \\
\hline \multicolumn{3}{|l|}{ Capacidade para exercer a profissão } \\
\hline Muito boa ou boa & 1,00 & \\
\hline Moderada ou baixa ou muito baixa & $1,28(1,08-1,52)$ & 0,004 \\
\hline \multicolumn{3}{|l|}{ Variáveis Proximais } \\
\hline \multicolumn{3}{|l|}{ Atividade física no tempo livre } \\
\hline Sim & 1,00 & \\
\hline Não & $1,66(1,38-2,00)$ & $<0,001$ \\
\hline \multicolumn{3}{|l|}{ Qualidade da alimentação autorreferida } \\
\hline Muito boa ou boa ou regular & 1,00 & \\
\hline Ruim ou muito ruim & $1,25(1,05-1,49)$ & 0,012 \\
\hline \multicolumn{3}{|l|}{ Índice de massa corporal } \\
\hline Baixo peso ou eutrófico & $1,22(1,05-1,43)$ & 0,009 \\
\hline Sobrepeso ou obesidade & 1,00 & \\
\hline $\begin{array}{l}\text { Variáveis incluídas no modelo final hierarquiza } \\
\text { para exercer a profissão; Nível Proximal: Ativid } \\
\text { alimentação autorreferida, tabagismo atual e ín }\end{array}$ & $\begin{array}{l}\text { aixa etária; Nível Interme } \\
\text { o livre, consumo excessiv } \\
\text { poral. }\end{array}$ & $\begin{array}{l}\text { egular e Capacidade } \\
\text { blicas, qualidade da }\end{array}$ \\
\hline
\end{tabular}

verduras e legumes elevados, mas apenas razoável consumo de frutas e retirada da gordura visível de carnes. Em adição, referiram baixo consumo de salgados fritos e doces, porém elevado consumo de bebidas açucaradas industrializadas. Também se detectou que mais de $50 \%$ dos motoristas apresentaram quatro ou mais comportamentos alimentares de risco à saúde, reforçando a necessidade de ações para promover mudanças desses comportamentos, especialmente com estímulo ao aumento da ingestão de frutas, ao consumo de carnes sem gordura visível, e à redução da ingestão de bebidas açucaradas industrializadas.

Este estudo verificou que a maioria das características de trabalho (Nível Intermediário) não apresentou relação com apresentar quatro ou mais comportamentos alimentares de risco à saúde. A exceção foi a percepção de pior capacidade para exercer a profissão de motorista, que apresentou associação com maior prevalência desses comportamentos. É possível que esta variável possa ser um importante indicador de sobrecarga de trabalho, o que contribui para piores comportamentos alimentares. Entretanto, deve-se mencionar que, apesar de longas jornadas de trabalho, turnos de trabalho irregulares e do cansaço ocasionado pelo exercício da profissão, a maioria dos pontos de parada propicia variedade de produtos alimentícios, sejam eles saudáveis ou não, de forma que as condições inadequadas para o exercício da profissão, aparentemente, não parecem ser importantes determinantes da escolha de alguns grupos alimentares.

No entanto, os resultados obtidos reforçam alguns achados observados na literatura. A identificação de indivíduos mais jovens com piores padrões alimentares foi verificada em outros estudos realizados no Brasil ${ }^{23-26}$, mas que se restringiram ao consumo de frutas, legumes e/ou hortaliças. O consumo alimentar mais adequado em indivíduos mais velhos pode estar associado a questões socioculturais, uma vez que os hábitos alimentares foram construídos em um momento no qual existia menor oferta de alimentos processados e industrializados, mas também pode estar associado a uma maior prevalência de doenças crônicas não transmissíveis entre os mais velhos, o que estimularia o consumo de alimentos mais saudáveis e a redução do consumo de alimentos deletérios à saúde ${ }^{25}$. 
Em relação à associação entre a inatividade física no tempo livre e um maior número de comportamentos alimentares de risco à saúde, também se verifica essa relação em outros estudos ${ }^{23,24,27,28}$. Estes comportamentos estão, de algum modo, associados ao contexto no qual as pessoas que adotam um estilo de vida mais ativo também tendem a ter hábitos alimentares mais saudáveis (e vice-versa) ${ }^{29}$. No que se refere ao IMC, percebeu-se relação contrária, ou seja, peso adequado associado ao maior número de comportamentos de risco à saúde, corroborando resultados de estudos de Dehghan et al. ${ }^{26} \mathrm{e}$ Lino et al..$^{30}$, mas contrariando estudo espanhol, o qual identificou que indivíduos com sobrepeso e obesos apresentaram maior consumo de frutas e vegetais ${ }^{31}$. Estudos transversais podem ter sua interpretação dificultada em relação à associação das variáveis testadas devido à possibilidade de viés de causalidade reversa, o que pode ter ocorrido no caso da relação contrária entre excesso de peso e menor frequência de consumo de alimentos de risco à saúde ${ }^{32}$.

Um resultado deste estudo que merece destaque é o maior número de comportamentos alimentares de risco à saúde em motoristas que referiram qualidade da alimentação ruim ou muito ruim. Esta relação denota que os motoristas têm consciência, de maneira geral, que sua alimentação não é saudável, o que reforça o argumento de que os comportamentos assumidos extrapolam o conhecimento do que se considera adequado ou inadequado. Assim, ações dirigidas a este grupo populacional e à população em geral não devem apenas se restringir à busca de redução de um determinado fator de risco à saúde, mas também visar mudanças comportamentais, as quais, na medida do possível, devem ser definidas em conjunto com o indivíduo, e não impostas de forma imperativa. Ademais, há de se considerar que o conhecimento muitas vezes é insuficiente para mudar comportamentos. Nesse sentido, o guia alimentar para a população brasileira representa um importante avanço, inclusive buscando uma compreensão mais abrangente, partindo do princípio de que a alimentação humana é muito mais do que a mera ingestão de nutrientes e que a adoção de uma alimentação saudável não é uma mera questão de escolha individual ${ }^{33}$. Destaca-se o reduzido número de motoristas que referiram retirar frequentemente a gordura antes de comer carne vermelha (menos de 50\%) e o elevado número que referiu consumir habitualmente bebidas açucaradas industrializadas (mais de 50\%). O Estudo Vigitel (2015) mostrou que 37,9\% dos homens avaliados referiram que costumam consumir carnes com excesso de gordura e 18,7\% relataram consumo de refrigerantes em cinco ou mais dias da semana. Em relação a frutas e hortaliças, $62,8 \%$ dos homens apresentaram consumo em cinco ou mais dias da semana ${ }^{34}$, resultado semelhante ao do presente estudo.

Os alimentos naturais ou minimamente processados (como frutas, verduras e legumes) costumam ser boas fontes de fibras e de vários nutrientes, além de terem baixo teor de calorias. Os de origem animal (carnes) são boas fontes de proteínas, vitaminas e minerais, porém, o teor excessivo de gorduras saturadas pode favorecer o risco de doenças cardiovasculares. Bebidas industrializadas ou refrigerantes são, normalmente, produtos ultra processados, com composição nutricional desbalanceada, ricos em açúcares, contribuindo para o desenvolvimento de obesidade, doenças cardiovasculares e diabetes mellitus ${ }^{33,35}$.

Observaram-se altos índices de sobrepeso/ obesidade $(81,1 \%)$ nos motoristas avaliados. Essa proporção é elevada quando comparada às observadas em estudos realizados com a população geral ${ }^{34,36}$. Em relação à inatividade física no tempo livre, vale mencionar que a frequência observada $(68,2 \%)$, apesar de elevada, mostra-se semelhante a achados de outros estudos de base populacional $^{37-39}$. A observação de elevadas proporções de excesso de peso, sedentarismo e de motoristas com quatro ou mais comportamentos alimentares de risco à saúde $(53,1 \%)$ reforça a necessidade de intervenções dirigidas a esses profissionais a fim de melhorar alguns dos comportamentos deletérios apresentados.

No Brasil, entre as 12 metas nacionais propostas pelo Plano de Ações Estratégicas para o Enfrentamento das Doenças Crônicas Não Transmissíveis (DCNT), duas estão diretamente relacionadas à alimentação saudável, especificamente: aumentar o consumo de frutas e hortaliças e reduzir o consumo médio de sal. Para tanto, um dos três eixos desse plano diz respeito à promoção da saúde, e dentro destes está a alimentação saudável, que, entre outras ações, propõe o aumento da oferta de alimentos saudáveis e a redução dos preços desses alimentos ${ }^{40}$. Já a Política Nacional de Promoção da Saúde, reformulada em 2014, coloca a alimentação adequada e saudável como um de seus oito temas prioritários ${ }^{41}$. Ainda assim, há desafios para a adoção de hábitos alimentares saudáveis, particularmente por uma categoria profissional que tem que se alimentar frequentemente fora de casa, como os trabalhadores do presente estudo. Para tanto, há de se considerar as especi- 
ficidades da profissão de motoristas de caminhão. No caso dos hábitos alimentares, é importante destacar que muitos se alimentam em pontos de parada e convivem em um contexto social de trabalho no qual a maioria dos profissionais tem hábitos alimentares prejudiciais à saúde ${ }^{17,42}$. Além disso, suas viagens podem durar vários dias ou semanas ${ }^{43}$, o que pode comprometer a qualidade de sua alimentação e dificultar ou impossibilitar a adequada e regular realização de atividade física no tempo livre. Em 2012 foi aprovada e entrou em vigor, no Brasil, a Lei Federal 12.619/12, conhecida como "Lei do Descanso", atualizada em $2015^{44}$, a qual regulamentou a jornada de trabalho diária e semanal dos motoristas de caminhão, além de definir regras para horas extras, hora noturna e tempo de espera para carga e descarga ${ }^{45}$. No entanto, são necessárias políticas públicas que estimulem a estruturação de locais de parada, inclusive por meio de incentivos fiscais.

Em adição, todo esse contexto implica a necessidade de adoção de estratégias de promoção à alimentação saudável, atividade física e redução do peso que não podem se restringir às estratégias utilizadas para a população geral, a qual geralmente tem sua residência como local de moradia, diferentemente do motorista de caminhão, que passa maior parte de seus dias e noites dentro do próprio veículo. Desse modo, as estratégias voltadas para esse subgrupo da população poderiam incluir o incentivo para que os pontos de parada, as transportadoras e os locais de carga e descarga tivessem estruturas para a realização de atividade física; que os restaurantes fomentassem uma alimentação saudável, por meio de cardápios específicos para esses profissionais; e de programas, nesses locais, de incentivo à redução de peso.

Algumas limitações metodológicas deste estudo merecem ser destacadas: a seleção da amos- tra foi por conveniência, o que não garante que represente o total de motoristas que frequentaram o Pátio de Triagem do Porto de Paranaguá no período de estudo; as informações se limitaram aos motoristas ativos, ou seja, motoristas afastados da profissão não foram incluídos; as informações foram obtidas por medidas indiretas, com a utilização de formulário de coleta, o qual, ainda que bem elaborado e aplicado por equipe bem treinada, está sujeito a vieses de memória, mensuração e informação. Por outro lado, os aspectos relacionados aos comportamentos alimentares foram adaptados de um instrumento já consolidado na literatura brasileira e utilizado no Estudo Vigitel ${ }^{21}$. Também, ainda que os dados de peso e altura tenham sido autorreferidos, o que tende a subestimar ou superestimar o valor desta medida, estudos têm demonstrado que essa diferença tende a ser pequena tanto em termos de $\mathrm{kg} /$ $\mathrm{m}^{2}{ }^{46}$ como em classificação categórica ${ }^{47}$.

\section{Considerações finais}

De forma geral, os resultados obtidos no presente estudo devem ser valorizados e analisados pelas autoridades e entidades representativas com o objetivo de implementar medidas visando à melhoria dos hábitos de vida, em especial dos comportamentos alimentares desses profissionais. Em adição, sugere-se que futuros estudos tenham abordagem qualitativa, buscando uma compreensão mais aprofundada sobre os comportamentos alimentares deste grupo de trabalhadores ou que procurem avaliar as barreiras percebidas para a adoção de uma alimentação mais saudável, além de estudos que busquem avaliar o impacto de intervenções voltadas à promoção de comportamentos alimentares mais saudáveis.

\section{Colaboradores}

E Girotto, SM Andrade e AE Mesas delinearam o estudo. E Girotto, CM Guidoni e MR Loch realizaram a análise estatística e escreveram o primeiro rascunho do manuscrito. AD González supervisionou as análises estatísticas e preparação do manuscrito. Todos os autores contribuíram para a elaboração e revisão do manuscrito e aprovaram a versão final do artigo submetido. 


\section{Referências}

1. Bjerregaard P. Nutritional transition - where do we go from here? J Hum Nutr Diet 2010; 23(Supl. 1):1-2.

2. Kac G, Velásquez-Meléndez G. A transição nutricional e a epidemiologia da obesidade na América Latina. Cad Saude Publica 2003; 19(Supl. 1):S5-S4.

3. Malta DC, Stopa SR, Iser BP, Bernal RT, Claro RM, Nardi AC, Reis AAC, Monteiro CA. Fatores de risco e proteção para doenças crônicas por inquérito telefônico nas capitais brasileiras, Vigitel 2014. Rev Bras Epidemiol 2015; 18(Supl. 2):238-255.

4. Malta DC, Bernal RTI, Nunes ML, Oliveira MM, Iser BPM, Andrade SSCA, Claro RF, Monteiro CA, Silva Júnior JB. Prevalência de fatores de risco e proteção para doenças crônicas não transmissíveis em adultos: estudo transversal, Brasil 2012. Epidemiol Serv Saúde 2014; 23(4):609-622.

5. Azevedo ECC, Diniz AS, Monteiro JS, Cabral PC. Padrão alimentar de risco para as doenças crônicas não transmissíveis e sua associação com a gordura corporal - uma revisão sistemática. Cien Saude Colet 2014; 19(5):1447-1458.

6. Confederação Nacional dos Transportes. Boletim Estatístico - CNT - Fevereiro 2017 [Internet]. Brasília: CNT. 2017 - [cited 2017 Nov 9]. Available from: http://www.cnt.org.br.

7. Silva LG, Luz AA, Vasconcelos SP, Moreno CRC. Vínculos empregatícios, condições de trabalho e saúde entre motoristas de caminhão. Rev Psicol Organ Trab 2016; 16(2):153-165.

8. Ulhoa MA, Marqueze EC, Lemos LC, Silva LG, Silva AA, Nehme P, Fischer FM, Moreno CR. Minor psychiatric disorders and working conditions in truck drivers. Rev Saude Publica 2010; 44(6):1130-1136.

9. Souza ER, Minayo MCS, Malaquias JV. Violência no trânsito: Expressão da violência social. In: Brasil. Ministério da Saúde (MS), editor. Impacto da violência na saúde dos brasileiros. Brasília: MS; 2005. p. 279-312.

10. Girotto E, Andrade SM, Gonzalez AD, Mesas AE. Professional experience and traffic accidents/near-miss accidents among truck drivers. Accid Anal Prev 2016; 95(Pt A):299-304.

11. Penteado RZ, Gonçalves CGO, Costa DD, Marques JM. Trabalho e saúde em motoristas de caminhão no interior de São Paulo. Saúde Soc. 2008; 17(6):35-45.

12. Takitane J, de Oliveira LG, Endo LG, de Oliveira KC, Munoz DR, Yonamine $\mathrm{M}$, et al. Uso de anfetaminas por motoristas de caminhão em rodovias do Estado de São Paulo: um risco à ocorrência de acidentes de trânsito?. Cien Saude Colet 2013; 18(5):1247-1254.

13. Ferreira SS, Alvarez D. Organização do trabalho e comprometimento da saúde: um estudo em caminhoneiros. Sist \& Gest [Internet]. 2013 Mar [cited 2017 Nov 9]; 8(1):58-66. Available from: http://www.revistasg.uff.br/index.php/sg

14. Oliveira LG, de Souza LM, Barroso LP, Gouvea MJ, de Almeida CV, Muñoz DR, Leyton V. Condições ocupacionais e o risco de uso de anfetaminas entre motoristas de caminhão. Rev Saude Publica 2015; 49:61.

15. Pylkkonen M, Sihvola M, Hyvarinen HK, Puttonen S, Hublin C, Sallinen M. Sleepiness, sleep, and use of sleepiness countermeasures in shift-working long-haul truck drivers. Accid Anal Prev 2015; 80:201-210.
16. Moreno CR, Carvalho FA, Lorenzi C, Matuzaki LS, Prezotti S, Bighetti P, Louzada FM, Lorenzi-Filho G. High risk for obstructive sleep apnea in truck drivers estimated by the Berlin questionnaire: prevalence and associated factors. Chronobiol Int 2004; 21(6):871879.

17. Codarin MAF, Moulatlet EM, Nehme P, Ulhôa M, Moreno CRC. Associação entre prática de atividade física, escolaridade e perfil alimentar de motoristas de caminhão. Saúde Soc. 2010; 19(2):418-428.

18. Puhkala J, Kukkonen-Harjula K, Aittasalo M, Mansikkamaki K, Partinen M, Hublin C, Kärmeniemi P, Sallinen M, Olkkonen S, Tokola K, Ojala A, Nygård $\mathrm{CH}$, Fogelholm M. Lifestyle counseling in overweight truck and bus drivers - Effects on dietary patterns and physical activity. Prev Med Rep 2016; 4:435-440.

19. Gilson ND, Pavey TG, Vandelanotte C, Duncan MJ, Gomersall SR, Trost SG, Brown WJ. Chronic disease risks and use of a smartphone application during a physical activity and dietary intervention in Australian truck drivers. Aust N Z J Public Health 2016; 40(1):91-93.

20. Balieiro LC, Rossato LT, Waterhouse J, Paim SL, Mota MC, Crispim CA. Nutritional status and eating habits of bus drivers during the day and night. Chronobiol Int 2014; 31(10):1123-1129.

21. Brasil. Ministério da Saúde (MS). Vigitel Brasil 2011: Vigilância de fatores de risco e proteção para doenças crônicas por inquérito telefônico. Brasília: MS; 2012.

22. Victora CG, Huttly SR, Fuchs SC, Olinto MT. The role of conceptual frameworks in epidemiological analysis: a hierarchical approach. Int J Epidemiol 1997; 26(1):224-227.

23. Figueiredo ICR, Jaime PC, Monteiro CA. Fatores associados ao consumo de frutas, legumes e verduras em adultos da cidade de São Paulo. Rev Saude Publica 2008; 42(5):777-785.

24. Neutzling MB, Rombaldi AJ, Azevedo MR, Hallal PC. Fatores associados ao consumo de frutas, legumes e verduras em adultos de uma cidade no Sul do Brasil. Cad Saude Publica 2009; 25(11):2365-2374.

25. Jaime PC, Figueiredo IC, Moura EC, Malta DC. Fatores associados ao consumo de frutas e hortaliças no Brasil, 2006. Rev Saude Publica 2009; 43(Supl. 2):5764.

26. Dehghan M, Akhtar-Danesh N, Merchant AT. Factors associated with fruit and vegetable consumption among adults. J Hum Nutr Diet 2011; 24(2):128-134.

27. Camoes M, Lopes C. Dietary intake and different types of physical activity: full-day energy expenditure, occupational and leisure-time. Public Health Nutr 2008; 11(8):841-848

28. Estaquio C, Druesne-Pecollo N, Latino-Martel P, Dauchet L, Hercberg S, Bertrais S. Socioeconomic differences in fruit and vegetable consumption among middle-aged French adults: adherence to the 5 A Day recommendation. J Am Diet Assoc 2008; 108(12):2021-2030.

29. Silva DA, Peres KG, Boing AF, Gonzalez-Chica DA, Peres MA. Clustering of risk behaviors for chronic noncommunicable diseases: a population-based study in southern Brazil. Prev Med 2013; 56(1):20-24. 
30. Lino MZ, Muniz PT, Siqueira KS. Prevalência e fatores associados ao excesso de peso em adultos: inquérito populacional em Rio Branco, Acre, Brasil, 2007-2008. Cad Saude Publica 2011; 27(4):797-810.

31. Agudo A, Pera G. Vegetable and fruit consumption associated with anthropometric, dietary and lifestyle factors in Spain. EPIC Group of Spain. European Prospective Investigation into Cancer. Public Health Nutr 1999; 2(3):263-271.

32. Agardh EE, Ahlbom A, Andersson T, Ostenson CG. The magnitude of bias in a cross-sectional study on lifestyle factors in relation to Type 2 diabetes. Scand J Public Health 2006; 34(6):665-668.

33. Brasil. Ministério da Saúde (MS). Guia alimentar para a população brasileira. Brasília: MS; 2014.

34. Brasil. Ministério da Saúde (MS). Vigitel Brasil 2015 Saúde Suplementar: vigilância de fatores de risco e proteção para doenças crônicas por inquérito telefônico. Brasília: MS; 2017.

35. Monteiro CA, Cannon G, Levy RB, Claro RM, Moubarac J-C. Ultra-processing and a new classification of foods. In: Neff R, editor. Introduction to the US Food System: Public Health, Environment, and Equity. San Francisco: Jossey Bass; 2015. p.338-339.

36. Holanda LGM, Martins MCC, Souza Filho MD, Carvalho CMRG, Assis RC, Leal LMM, Mesquita LPL, Costa EM. Excesso de peso e adiposidade central em adultos de Teresina-PI. Rev Assoc Med Bras 2011; 57(1):50-55.

37. Romeiro-Lopes TC, Franca-Gravena AA, Dell Agnolo CM, Rocha-Brischiliari SC, Barros Carvalho MD, Pelloso SM. Fatores associados à inatividade física no lazer em município do Sul do Brasil. Rev Salud Pública 2014; 16(1):40-52.

38. Loch MR, Bortoletto MSS, Tanno de Souza RK, Mesas AE. Simultaneidade de comportamentos de risco para a saúde e fatores associados em estudo de base populacional. Cad Saúde Colet 2015; 23(2):180-187.

39. Knuth AG, Malta DC, Dumith SC, Pereira CA, Morais Neto OL, Temporão JG, Penna G, Hallal PC. Prática de atividade física e sedentarismo em brasileiros: resultados da Pesquisa Nacional por Amostra de Domicílios (PNAD) 2008. Cien Saude Colet 2011; 16(9):3697-3705.

40. Malta DC; Silva Junior JB. O Plano de ações estratégicas para o enfrentamento das doenças crônicas não transmissíveis no Brasil e a definição das metas globais para o enfrentamento dessas doenças até 2025: uma revisão. Epidemiol Serv Saúde 2013; 22(1):151164.

41. Brasil. Ministério da Saúde (MS). Política Nacional de Promoção da Saúde: PNaPS: revisão da Portaria MS/ $G M n^{\circ}$ 687, de 30 de março de 2006. Brasília: MS; 2014.

42. Masson VA, Monteiro MI. Estilo de vida, aspectos de saúde e trabalho de motoristas de caminhão. Rev Bras Enferm 2010; 63(4):533-540.

43. Knauth DR, Pilecco FB, Leal AF, Seffner F, Teixeira AM. Manter-se acordado: a vulnerabilidade dos caminhoneiros no Rio Grande do Sul. Rev Saude Publica 2012; 46(5):886-893.
44. Brasil. Lei $\mathrm{n}^{\circ}$ 13.103, de 2 de março de 2015. Dispõe sobre o exercício da profissão de motorista; altera a Consolidação das Leis do Trabalho - CLT, aprovada pelo Decreto-Lei no 5.452, de 1 o de maio de 1943, e as Leis nos 9.503, de 23 de setembro de 1997 - Código de Trânsito Brasileiro, e 11.442, de 5 de janeiro de 2007 (empresas e transportadores autônomos de carga), para disciplinar a jornada de trabalho e o tempo de direção do motorista profissional; altera a Lei no 7.408, de 25 de novembro de 1985; revoga dispositivos da Lei no 12.619, de 30 de abril de 2012; e dá outras providências. Diário Oficial da União 2015; 2 mar.

45. Brasil. Lei no 12.619 , de 30 de abril de 2012. Dispõe sobre o exercício da profissão de motorista. Diário Oficial da União 2012; 30 abr.

46. Niedhammer I, Bugel I, Bonenfant S, Goldberg M, Leclerc A. Validity of self-reported weight and height in the French GAZEL cohort. Int J Obes Relat Metab Disord 2000; 24(9):1111-1118.

47. Carvalho AM, Piovezan LG, Selem SS, Fisberg RM, Marchioni DM. Validation and calibration of self-reported weight and height from individuals in the city of São Paulo. Rev Bras Epidemiol 2014; 17(3):735-746.
Artigo apresentado em 15/12/2017

Aprovado em 05/07/2018

Versão final apresentada em 07/07/2018 
